\title{
Utilización de WhatsApp para la Comunicación en Titulados Superiores
}

\author{
WhatsApp Use for Communication among Graduates
}

\author{
María-Teresa Gómez-del-Castillo * \\ Universidad de Sevilla
}

\begin{abstract}
En esta investigación nos planteamos conocer qué uso lingüístico hacen de WhatsApp las personas con estudios universitarios, y estudiar las posibilidades de incorporar este tipo de escritura en los centros educativos. Trabajamos con una muestra de 38 personas con estudios superiores, analizando 529 conversaciones y 3.872 intervenciones de WhatsApp. Los resultados nos permiten afirmar que la comunicación por mensajería instantánea ha dejado de ser exclusivamente de tipo textual, y cada vez se hace más expresiva y variada con imágenes, vídeos, audios, stickers o referencias a webs. Este uso multimediático de la mensajería se ha impuesto, también, en hablantes con niveles de estudios elevados. Vemos como los usuarios más jóvenes realizan conversaciones más largas que los mayores, y las mujeres más extensas que los hombres, pero no se han encontrado diferencias significativas entre los sujetos de distintas titulaciones. El uso de disonancias con la norma culta de escritura se da más frecuentemente entre los sujetos más jóvenes y/o con menor nivel de estudios que en los graduados; lo mismo ocurre con el uso de archivos intercalados en la conversación y con la utilización de emoticonos. También comprobamos que la incorporación de los teléfonos inteligentes a los procesos de enseñanza-aprendizaje (m-learning) está siendo cada vez más importante.
\end{abstract}

Descriptores: Aprendizaje en línea, Tecnología educacional, Aprendizaje informal, Educación.

In this research, we consider the linguistic use of WhatsApp made by people with university studies, and to study the possibilities of incorporating this type of writing in schools. We worked with a sample of 38 people with higher education, analyzing 529 conversations and 3,872 WhatsApp interventions. The results allow us to affirm that communication by instant messaging is no longer exclusively textual, and it is becoming more expressive and varied with images, videos, audios, stickers or references to websites. This multimedia messaging usage has also been imposed on speakers with high levels of study. We see how younger users engage in longer conversations than older ones, and women, in longer conversations than men, but no significant difference has been found between subjects from different academic degrees. The use of dissonances with the cultured writing norms occurs more frequently among the younger subjects and / or with lower level of studies than in the graduates. The same happens with the use of files interspersed in the conversation and with the use of emoticons. We also see that the incorporation of smartphones into the processes of teaching and learning (m-learning) is becoming increasingly important.

Keywords: Electronic learning, Educational technology, Informal learning, Education.

Investigación financiada dentro del Proyecto de Investigación "Mensajería Instantánea y Aprendizaje” del Grupo de Investigación Didáctica de la Junta de Andalucía: Código HUM-39o

*Contacto: mgomezdelcastillo@us.es

ISSN: $1696-4713$

www.rinace.net/reice/

revistas.uam.es/reice
Recibido: $\quad 15$ de abril 2017

$1^{\text {a }}$ Evaluación: 21 de mayo 2017

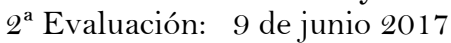

Aceptado: $\quad 30$ de junio 2017 


\section{Comunicación digital interpersonal}

Como sabemos el WhatsApp es un servicio de mensajería instantánea cuyo propósito es comunicar, vía Internet y en tiempo real, a dos o más usuarios entre sí con un mínimo coste económico y empleando mensajes de texto o archivos (audios, videos, imágenes, ubicación geográfica...). Es una aplicación que se puede instalar en todos los smartphones o teléfonos inteligentes (Calero, 2014), que se ha convertido en el sistema mundial de comunicación por excelencia entre teléfonos móviles, a través de datos o por wifi.

A comienzos de 2016 Facebook informó de que su app WhatsApp superó los mil millones de usuarios activos en el mundo. En los últimos tiempos ha ido evolucionando (no cobrando por sus servicios o incorporando las llamadas de voz), y hasta ahora no ha sido superada ni siquiera por sus cercanos competidores como Line o WeChat. Su desarrollo futuro parece encaminado a convertirse en un portal de contenidos para responder a las demandas de las corporaciones (Hernández, 2016).

En España, el dispositivo más utilizado para la conexión a internet es el Smartphone según la Encuesta General de Medios de abril y mayo de 2016 con un 93,6\% de los usuarios de la red, que en nuestro país suponen casi el $76 \%$ de la población. Prácticamente todos se conectan desde sus casas y aumentan considerablemente los usuarios que se conectan en la calle o en los transportes (desde el $24 \%$ en 2013 al 59,2\% en la actualidad) (Asociación para la Investigación de Medios de Comunicación, 2016).

Cremades, Maqueda y Onieva (2016) consideran que la aplicación WhatsApp es un modo evolucionado de comunicación escrita caracterizado por la posibilidad de enriquecer el discurso con contenidos audiovisuales e icónicos. Otros lo consideran como un acercamiento del lenguaje escrito al lenguaje natural, más propio de la oralidad (que en español es muy parecido, no así en inglés) completado semióticamente con imágenes o gestos (Calero, 2014; Sampietro, 2016). Aunque este tipo de comunicación no es incompatible con el uso culto del lenguaje escrito, el uso "oralizado" que en la práctica se hace de él ha hecho que algunas instituciones manifiesten su inquietud al respecto y pidan al sistema educativo que garantice el aprendizaje de la norma culta en la escritura.

Una de las características de los lenguajes digitales es que facilitan los encuentros interpersonales, sin que el lugar y el horario de los que se comunican sea una limitación importante (Iniciarte, 2004). El usuario puede comunicarse con cualquiera en cualquier parte del mundo y en cualquier momento, aunque es cierto que, a diferencia de otros medios digitales, la comunicación por WhatsApp suele requerir un conocimiento mutuo (más o menos cercano) entre los emisores-receptores de los mensajes, ya que la agenda de contactos forma parte de la red de conocidos. A diferencia de los foros u otro tipo de chat, en WhatsApp la identidad del interlocutor es real. No obstante, no es menos cierto que con estos medios han aparecido nuevos comportamientos socio-comunicativos que han generado lo que algunos sociólogos denominan "falsa extimidad", donde algunas veces la comunicación es más con personajes que con personas (Galán, 2011).

Otra característica es que el intercambio textual es rápido y espontáneo, intentando demostrar al interlocutor, en muchas ocasiones, que se está conectado de manera permanente. La inmediatez es tan importante que la comunicación no es ya un intercambio de información sino un objeto de consumo, llegándose, en algunos casos, a la adicción de la interconexión permanente, muy común entre sujetos de todas las edades, pero especialmente entre la población joven (Morduchowicz, 2013). 
En WhatsApp las normas de sintaxis u ortografía pasan a un segundo plano, lo prioritario es transmitir los mensajes con prontitud y eficacia, ya sea con textos, símbolos, imágenes o audios (Galán, 2011). Cada vez más jóvenes prefieren comunicarse a través de estas redes que hacerlo personalmente, sin tener en cuenta que puede generarse el peligro de volverse dependientes y de encerrarse en un mundo donde la tecnología domina.

\section{Importancia social del WhatsApp}

Rubio y Perlado (2015) analizan el fenómeno WhatsApp en los jóvenes universitarios y justifican su uso porque, dicen, es cómodo y práctico. Y añaden que resulta incluso paradójico cómo, "teniendo en cuenta que los smartphones permiten la posibilidad de usar mensajes de voz en lugar de escritos, esta función apenas aparezca en los discursos de los jóvenes” (p. 81). Estos autores señalan que, aunque la comunicación digital es fría, es utilizada continuamente debido a los ritmos impuestos en la vida diaria. Reconocen la trivialidad de gran cantidad de la información enviada, así como que es más usual que la comunicación presencial, que se reserva para contenidos más personales. Sin embargo, resulta paradójico que reconozcan que las personas no se comunican más que antes, sino que lo hacen de otra manera.

Villamandos (2016) nos invita a reflexionar sobre la importancia de esta aplicación, repasando algunos de los datos más curiosos asociados a sus mil millones de usuarios:

- Se intercambian 42.000 millones de mensajes diarios, que supone una media de 42 mensajes diarios por usuario.

- Según los datos de WhatsApp existen mil millones de grupos lo que hace que toque a una moderación por usuario.

- La empresa está compuesta por 57 ingenieros, uno por cada 17,5 millones de personas que usan WhatsApp.

- WhatsApp habla en 53 idiomas. La India, Sudáfrica, Singapur y Hong Kong tienen una cuota de penetración por encima del $70 \%$.

- Facebook compra la aplicación por 21.800 millones, después de haber competido con Google.

Estas son algunas de las cifras del fenómeno WhatsApp, y las previsiones de futuro indican que seguirán aumentando y, por tanto, aumentando su impacto social.

\subsection{Perspectiva socio-lingüística}

Partiendo de la necesitad del aprendizaje de las competencias lingüísticas, Blanch, Betancort y Martínez (2016) distinguen tres bloques de destrezas necesarias en la población para la comunicación a través de medios como WhatsApp: a) la compresión del contenido de los medios, que incluye un aprendizaje para interpretar los formatos multimedia; b) la evaluación crítica de la información, que hace necesaria la capacidad para discernir su veracidad y la expresión lingüística utilizada; y c) el acceso eficaz a la información y producción de mensajes, que incluye la capacidad de uso de las TIC y el empleo del lenguaje según el medio utilizado. 
El uso que se hace del lenguaje en las redes sociales en general, y en WhatsApp en particular, es muy característico. Diversos autores lo han estudiado en los últimos años encontrado que en estos soportes no son frecuentes los textos extensos en escritura estándar (Cassany y Sanz, 2011; Gómez-Camacho y Gómez-del-Castillo, 2013). Puesto que el símbolo posee la capacidad de transmitir emociones con más rapidez y eficacia que las palabras, la escritura se hace gráfica o icónica, que es un tipo de escritura simbólica más propia del lenguaje no verbal y de las relaciones cercanas o familiares. El valor de la imagen es su capacidad para transmitir una información que no puede codificarse de otra forma, por eso, se utilizan con frecuencia, cuando se quieren expresar sentimientos o ideas de forma inmediata (Calero, 2014). El uso de emoticonos dentro del mensaje, la repetición de emoticonos exclusivamente, el uso de imágenes o de audios en él y el uso de videos como parte de la conversación son los elementos multimedia más utilizados (Gómez-Camacho y Gómez-del-Castillo, 2015).

Diversas investigaciones realizadas sobre adolescentes (Vázquez-Cano, Mengual-Andrés y Roig-Vila, 2015) o sobre estudiantes universitarios (Gómez-Camacho y Gómez-delCastillo, 2015) muestran cómo la mayoría de los usuarios de WhatsApp se alejan de la norma culta, en mayor o menor grado, al comunicarse con este medio. Las repeticiones enfáticas de los elementos finales de palabra (guapaaaa...), la repetición de emoticonos y signos de cierre de interrogación y admiración (jajaja, :-) :-) :-)...), la supresión de letras (tb, tngo...) y sílabas (mates...), el uso de siglas no normativas (q, x...), grafemas no normativos (kien, xq...), textismos léxicos (sip, jartao, pos...) así como un uso de las mayúsculas sujeto a normas ajenas a la tradición culta del español son rasgos que definen la escritura de los mensajes de texto en español, comunes a cualquier usuario independientemente del nivel cultural. Este uso disortográfico del lenguaje también ha sido descrito en los trabajos de Bernicot y colaboradores (2014) para el francés, y los de Gann, Bartoszuk y Anderson (2010) y Powell y Dixon (2011) para el inglés, no encontrando relaciones significativas entre las dificultades ortográficas de los sujetos estudiados y las discrepancias intencionadas con la norma académica en los mensajes de WhatsApp.

\subsection{Perspectiva socio-educativa}

Las nuevas maneras de escribir al margen de la norma culta se incorporan inevitablemente al mundo educativo dentro o fuera del curriculum oficial, dándose una transferencia de este modo de usar el lenguaje desde el ámbito tecnológico al académico por parte de alumnos y profesores. Algunas experiencias educativas (Díaz-Jatuf, 2014) ponen de manifiesto cómo las redes sociales como Facebook, Twitter o WhatsApp están penetrando en los centros educativos. Especialmente WhatsApp tiene una relevancia importante dado que profesores, familias y estudiantes, en cualquier parte del mundo, lo usan para comunicarse, enviar materiales de apoyo, test, imágenes educativas, información de todo tipo, de temáticas curriculares y extracurriculares.

Bouhnik y Deshen (2014) afirman que estar en contacto permanente tanto con maestros como entre compañeros mejora la comunicación educativa. La comunicación y el intercambio de textos, imágenes o vídeos de manera instantánea favorece la interactividad y la cooperación entre grupos que comparten trabajos, tareas o proyectos (Oliveira, Versuti y Vaz, 2016). El maestro, en la medida en que también puede enviar tareas o información, favorece el aprendizaje colaborativo, realiza pequeñas tutorías e incluso puede evaluar a través del propio WhatsApp (Padrón, 2013). En esta línea, hay 
documentadas experiencias del uso de esta tecnología en el aprendizaje de la escritura en lengua extranjera (Martínez-Parejo, 2016), o para mejorar el lenguaje hablado cuando la vergüenza, el miedo o cualquier otra razón hacen retraerse al alumno creando ansiedad y reduciendo su producción oral (Han y Keskin, 2016).

En esta misma línea, también se ha observado que existe un elemento de igualación en el uso de estos medios entre alumnos más o menos competentes lingüísticamente porque permite mayor flexibilidad y libertad en escritura (Gómez-Camacho y Gómez-delCastillo, 2015), suponiendo un elemento motivador para sujetos con menos habilidades lingüísticas. Por otra parte, se ha comprobado que los estudiantes alternan la escritura, con naturalidad, entre la norma culta y la norma disortográfica, por lo que condenar este tipo de textos o excluirlos del currículo de las áreas lingüísticas en los diferentes niveles educativos nos parece incongruente y contraproducente (Gómez-Camacho y Gómez-delCastillo, 2015). Creemos que su incorporación al currículum debe hacerse de forma explícita.

\section{Algunos problemas en el uso de WhatsApp}

Los usuarios de WhatsApp no pueden olvidar la falta de intimidad que nos ofrece, y la facilidad para hacerse con la información que se envía por este medio en cualquiera de sus formatos: textos, imágenes, audios o videos. Este es un primer inconveniente de WhatsApp, a saber, que los mecanismos de privacidad no están suficientemente conseguidos y es relativamente fácil que un tercero no deseado pueda acceder a la información.

En segundo lugar, debemos tener en cuenta que a veces el uso de smarphone nos acerca a quienes están lejos, pero nos aleja de quienes tenemos cerca (Clairá, 2013). Estamos conectados de forma permanente a internet, y esto ha llegado a convertirse en una necesidad social y laboral llegando, en algunos casos, a provocar ansiedad, insomnio, adicciones tecnológicas u otras disfunciones sociales. Muestra de ello es la emergencia de la expresión phubbing, formado a partir de las palabras phone (teléfono) y snubbing (despreciar), para referirse a las interrupciones en interacciones cara a cara sufridas en reuniones de amigos o de trabajo, como consecuencia de la conectividad permanente de algunas personas (Capilla y Cubo, 2017).

Por último, han sido referenciados trastornos psicológicos como consecuencia del uso de aplicaciones como WhatsApp (Giménez y Zirpoli, 2015), son los siguientes:

- El síndrome de la llamada imaginaria: imaginar que su teléfono inteligente ha sonado o vibrado cuando no lo ha hecho.

- La nomofobia: angustia que el usuario experimenta cuando no tiene acceso al teléfono.

- La dependencia de Internet: necesidad de estar constantemente conectados a la red, llegando a establecerse vínculos adictivos que pueden dañar la vida privada y las relaciones sociales.

- Síndrome del doble check: ansiedad que sufre el sujeto emisor al ver que el destinatario no responde, sabiendo que el mensaje le ha llegado y que ha estado conectado después de recibir el WhatsApp. 


\section{Método}

Partiendo del interés creciente hacia el mobile learning, y teniendo en cuenta que puede ser implementado en cualquier etapa educativa, con este trabajo queremos contribuir a la reflexión sobre el uso de la aplicación WhatsApp, dado que es, en estos momentos, una herramienta muy familiar para cualquier miembro de la comunidad educativa (niños, jóvenes, padres, profesores...), y que permite tener una comunicación personal o grupal en tiempo real.

En concreto queremos recoger información relacionada con la utilización de esta aplicación, pretendiendo: conocer qué uso lingüístico hacen del WhatsApp las personas con estudios universitarios; y estudiar las posibilidades de incorporar este tipo de escritura en los centros educativos como objetivo del currículum dentro de la competencia digital y de las competencias lingüísticas.

El enfoque de investigación de este estudio es experimental y combina una metodología cuantitativa y cualitativa, en cuanto que hacemos un análisis de contenidos (Valencia y García, 2010), seleccionando y analizando diversos elementos de los textos de WhatsApp recibidos, intentando contribuir, con nuestra aportación, a la descripción de las experiencias de aquellos que se enfrentan al estudio de estos medios. No pretendemos establecer principios que expresen relaciones de causalidad, sino que pretendemos descubrir patrones que permitan comprender el sentido y las características de este fenómeno social.

En cuanto al diseño de investigación podríamos concretarlo en un estudio de casos amplio, centrando las experiencias en personas adultas con estudios superiores terminados y que en su totalidad utilizan la aplicación WhatsApp para comunicarse, sin pretender que los resultados obtenidos sean generalizables. Se trata de un estudio descriptivo, donde los contenidos seleccionados se han cuantificado, y hemos procesado dichos datos para extraer porcentajes y poderlos comparar, teniendo en cuenta, especialmente, la frecuencia de apariciones para posteriormente redactar los resultados a partir de un tratamiento estadístico (López, 2002).

La población sobre la que hemos hecho el estudio haría referencia a los titulados superiores españoles usuarios de WhatsApp a través de sus smartphones. Nuestra muestra está constituida por 38 personas con un grado universitario en titulaciones diversas (ingenierías, historia, bellas artes, enfermería, psicología, matemáticas, etc.), que en la actualidad están matriculadas en el Master Universitario en Profesorado de Enseñanza Secundaria Obligatoria y Bachillerato, Formación Profesional y Enseñanza de Idiomas (MAES) de la Universidad de Sevilla (España). De ellas 20 son varones y 18 son mujeres. La edad está comprendida entre los 23 y los 46 años.

De estos participantes analizamos 529 conversaciones de WhatsApp, 3.872 intervenciones y 20.404 palabras. Hay que tener en cuenta que todas las conversaciones analizadas se producen entre los sujetos de nuestra muestra (un estudiante del MAES) y otra persona de la que desconocemos su nivel de estudios y de las que, por tanto, rehusamos analizar sus mensajes, para centrarnos únicamente en los de las 38 personas anteriormente descritas.

Antes de continuar señalar que cuando hacemos referencia a conversaciones nos estamos refiriendo al diálogo en torno a un mismo tema, y que normalmente se produce de 
manera ininterrumpida en el tiempo. Produciéndose normalmente una nueva conversación cuando se da un salto temporal. La intervención se produce cada vez que se envía un mensaje. Es muy frecuente que la misma persona tenga varias intervenciones seguidas antes de dar paso a la respuesta del interlocutor (cuadro 1).

Cuadro 1. Distribución de la muestra por sexos

\begin{tabular}{|c|c|c|c|c|}
\hline & SUJETOS & \% SUJETOS & CONVERSACIONES & \% CONVERSAC. \\
\hline Hombres & 20 & $53 \%$ & 299 & $57 \%$ \\
\hline Mujeres & 18 & $47 \%$ & 230 & $43 \%$ \\
\hline \multirow[t]{2}{*}{ Total } & 38 & $100 \%$ & 529 & $100 \%$ \\
\hline & INTERVENCIONES & \% INTERVENCIONES & PALABRAS & \% PALABRAS \\
\hline Hombres & 1.869 & $48 \%$ & 9.562 & $47 \%$ \\
\hline Mujeres & 2.003 & $52 \%$ & 10.841 & $53 \%$ \\
\hline Total & 3.872 & $100 \%$ & 20.404 & $100 \%$ \\
\hline
\end{tabular}

Fuente: Elaboración propia.

Se han analizado más hombres que mujeres, tanto en sujetos como en conversaciones, y, sin embargo, tanto el número de intervenciones como el de palabras, es mayor en el caso de las mujeres que el de los hombres.

La distribución porcentual de la muestra según la edad se presenta en el cuadro 2 , y en el podemos observar cómo los alumnos menores de 34 años suponen el $74 \%$ de la muestra, frente a los mayores de esa edad que ascienden al $26 \%$. También podemos comprobar cómo los más jóvenes emplean más la aplicación que los más veteranos.

Cuadro 2. Distribución de la muestra por edades

\begin{tabular}{llcccc}
\hline & N & \% DE SUJETOS & \% CONVERSACIONES & \% INTERVENCIONES & \% PALABRAS \\
\hline 23-33 años & 28 & $74 \%$ & $75 \%$ & $86 \%$ & $84 \%$ \\
$34-46$ años & 10 & $26 \%$ & $25 \%$ & $14 \%$ & $16 \%$ \\
Total & 38 & $100 \%$ & $100 \%$ & $100 \%$ & $100 \%$ \\
\hline
\end{tabular}

Fuente: Elaboración propia.

Si nos fijamos en el tipo de titulación de los sujetos que componen la muestra, comprobamos que los graduados en carreras tradicionalmente consideradas como "de letras" en las que se incluyen las humanidades, las ciencias sociales y las artísticas, superan a los sujetos de titulaciones más relacionadas con las ciencias puras, las ciencias aplicadas, o las ciencias de la vida y de la salud, según se muestra en el cuadro 3.

Cuadro 3. Distribución de la muestra por titulaciones

\begin{tabular}{lccccc}
\hline & N & \% DE SUJETOS & \% CONVERSACIONES & \% INTERVENCIONES & \% PALABRAS \\
\hline Letras & 23 & $61 \%$ & $53 \%$ & $64 \%$ & $63 \%$ \\
Ciencias & 15 & $39 \%$ & $47 \%$ & $36 \%$ & $37 \%$ \\
Total & 38 & $100 \%$ & $100 \%$ & $100 \%$ & $100 \%$ \\
\hline
\end{tabular}

Fuente: Elaboración propia.

$\mathrm{El}$ instrumento para la recogida de los datos fueron los textos de WhatsApp producidos por los componentes de la muestra. La labor de los alumnos del MAES que colaboraron en esta investigación cediéndonos sus discursos digitales fue fundamental, y resulta prácticamente la única posibilidad de contar con muestras de textos amplias para poder 
trabajar con datos primarios y fiables, datos que no resultan fáciles de obtener (Vela y Cantamutto, 2016).

Como quisimos trabajar con sujetos de alto nivel cultural y de estudios, el procedimiento fue acudir al Master que, en España, forma al profesorado de Enseñanzas Medias, donde, para acceder a él, todos los alumnos deben ser titulados superiores. Nos dirigimos a un módulo común para encontrar en sus aulas alumnos de todas las titulaciones. Después de explicar cuál era nuestra trayectoria investigadora en el ámbito de la comunicación digital (redes sociales, mensajería instantánea,), les propusimos su participación en la investigación.

Acordamos que eligiesen una conversación personal de WhatsApp con algún amigo o compañero (no conversación de grupo), y la enviasen a nuestro correo electrónico en formato texto para poderla analizar con el programa ATLAS.ti y la hoja de cálculo Excel. Aunque al recibir el archivo aparece el nombre del emisor, enseguida borramos todas las referencias personales para trabajar con la información de manera anónima. Como hemos descrito en la muestra, las aportaciones recibidas fueron muy diversas en cuanto a la edad de los sujetos, el sexo o la titulación; datos que les solicitamos que escribieran en el último mensaje del archivo antes de enviarlo.

Hemos trabajado con un máximo de 20 conversaciones por cada persona y el resto del texto recibido lo hemos descartado, analizando solo las aportaciones realizadas por el alumno del MAEs, es decir, por la persona que sabíamos que poseía una titulación universitaria, descartando las aportaciones del otro interlocutor. Fuimos señalando las conversaciones, las intervenciones de nuestros sujetos de estudio, el número de palabras empleadas para ellas, los emoticonos y stickers, los archivos de imagen, audio o vídeo, los textismos, enlaces Web...etc.

Posteriormente procesamos estos datos, separando, con la ayuda de una hoja de cálculo, cada una de las palabras y otros elementos de las conversaciones para poderlas analizar y clasificar. Con todo ello sacamos los resultados que a continuación se presentan.

\section{Resultados}

En relación con nuestros objetivos vamos a comenzar la presentación de los resultados examinando la longitud de las conversaciones e intervenciones. Las conversaciones analizadas varían entre un mínimo de dos intervenciones y un máximo de 87 intervenciones, siendo la media por conversación de 16 intervenciones. Hemos de tener en cuenta que este número de intervenciones se refieren a ambos interlocutores, por lo que no coinciden con las intervenciones analizadas que han sido solo las emitidas por los sujetos de nuestra muestra. Ello significa que vamos a estudiar una media de 8 intervenciones por conversación.

En cuanto al número de palabras, encontramos una media de 5,3 palabras por intervención, lo que implica que cada conversación analizada supone 86 palabras aproximadamente. Teniendo en cuenta la diferencia por sexos, la extensión de la conversación ha resultado similar, de 5,1 en el caso de los hombres y 5,4 en el de las mujeres. Sin embargo, parece que sí son más largas las conversaciones de las mujeres que la de los hombres. Los varones analizados intervienen 6,3 veces en una conversación frente a las 8,7 veces que lo hacen nuestra muestra femenina (cuadro 4). 
Cuadro 4. Conversaciones, intervenciones y palabras analizadas por sexos

\begin{tabular}{lccccc}
\hline & CONVERS. & INTERVENC. & INTERV./CONV. & PALABRAS & PALAB./INTERV. \\
\hline Hombres & 299 & 1.869 & 6,3 & 9.562 & 5,1 \\
Mujeres & 230 & 2.003 & 8,7 & 10.841 & 5,4 \\
Total & 529 & 3.872 & & 20.404 & \\
\hline
\end{tabular}

Fuente: Elaboración propia.

El número de palabras por intervención si comparamos los sujetos por edad han resultado diferentes, los sujetos de 34 años o más escriben 6,2 palabras por intervención frente a los 5,1 de los más jóvenes (cuadro 5). Y sin embargo son más largas las conversaciones de los jóvenes (8,4 intervenciones por conversación) que la de los mayores ( 4,1 intervenciones por conversación). Estos últimos economizan más en cuanto al número de intervenciones, omitiendo frases innecesarias.

Cuadro 5. Conversaciones, intervenciones y palabras analizadas por edades

\begin{tabular}{lccccc}
\hline & CONVERS. & INTERVENC. & INTERV./CONV. & PALABRAS & PALAB./INTERV. \\
\hline 23-33 años & 399 & 3.343 & 8,4 & 17.098 & 5,1 \\
34-46 años & 130 & 529 & 4,1 & 3.306 & 6,2 \\
Total & 529 & 3.872 & & 20.404 & \\
\hline
\end{tabular}

Fuente: Elaboración propia.

Si nos fijamos en las palabras por intervención que utilizan los grupos diferenciados por tipo de titulación han resultado similares, entre 5,2 en el caso de los titulados de "letras" frente al 5,4 de los titulados en "ciencias", y en este caso son más largas las conversaciones de los que poseen titulaciones de "letras" (9 intervenciones por conversación) que los que la poseen en "ciencias" (5,5 intervenciones por conversación). Estos últimos economizan más en cuanto al número de veces que intervienen en una conversación, ahorrando en frases que consideran innecesarias (cuadro 6). En nuestro propósito de conocer el uso lingüístico que hacen del WhatsApp las personas con estudios universitarios, analizamos el número de emoticones, archivos y textismos que incluyeron en sus intervenciones (cuadro 7).

Cuadro 6. Conversaciones, intervenciones y palabras analizadas por titulaciones

\begin{tabular}{lccccc}
\hline & CONVERS. & INTERVENC. & INTERV./CONV. & PALABRAS & PALAB./INTERV. \\
\hline Letras & 278 & 2.491 & 9,0 & 12.913 & 5,2 \\
Ciencias & 251 & 1.381 & 5,5 & 7.491 & 5,4 \\
Total & 529 & 3.872 & & 20.404 & \\
\hline
\end{tabular}

Fuente: Elaboración propia.

Si nos fijamos en la primera línea que refleja los totales de la muestra, podemos destacar que el porcentaje de textismos en relación con el número de palabras es del 17,6\%. También podemos observar que el porcentaje de emoticonos empleados en relación al número de palabras es del 1,36\%. Algo mucho más significativo ocurre si observamos el uso de archivos (imágenes, video o sonido) en las conversaciones, donde una de cada tres incorpora algunos archivos. Si los relacionamos con el número de palabras los archivos suponen el $0,8 \%$. Observando el cuadro 8 , el empleo de emoticonos vemos que las diferencias en ninguna de las variables (sexo, edad, titulación) resultan relevantes. Puntúan de manera poco coherente, según nos fijemos en los emoticonos por conversación o por intervención. 
Cuadro 7. Emoticonos, archivos y textismos por sexo, edad y titulación

\begin{tabular}{lcccccc}
\hline & CONVERSAC. & INTERVENC. & PALABRAS & EMOTIC. & ARCHIVOS & TEXTISMOS \\
\hline Hombres & 299 & 1.869 & 10841 & 145 & 98 & 1438 \\
Mujeres & 230 & 2.003 & 9562 & 133 & 68 & 2155 \\
23-33 años & 399 & 3.343 & 17.098 & 216 & 120 & 3213 \\
$34-46$ años & 130 & 529 & 3.306 & 62 & 46 & 380 \\
Letras & 278 & 2.491 & 12.913 & 161 & 84 & 2585 \\
Ciencias & 251 & 1.381 & 7.491 & 117 & 82 & 1008 \\
Total & 529 & 3.872 & 20404 & 278 & 166 & 3593 \\
\hline
\end{tabular}

Fuente: Elaboración propia.

Cuadro 8. Porcentajes del uso de emoticonos y archivos en conversaciones e intervenciones por sexo, edad y titulación

\begin{tabular}{lcccc}
\hline & $\begin{array}{c}\text { CONVERSACIÓN } \\
\text { CON EMOTICONO }\end{array}$ & $\begin{array}{c}\text { INTERVENCIÓN } \\
\text { CON EMOTICONO }\end{array}$ & $\begin{array}{c}\text { CONVERSACIÓN } \\
\text { CON ARCHIVO }\end{array}$ & $\begin{array}{c}\text { INTERVENCIÓN } \\
\text { CON ARCHIVO }\end{array}$ \\
\hline Hombres & $48,5 \%$ & $7,8 \%$ & $32,8 \%$ & $5,2 \%$ \\
Mujeres & $57,8 \%$ & $6,6 \%$ & $29,6 \%$ & $3,4 \%$ \\
$23-33$ años & $54,1 \%$ & $6,5 \%$ & $30,1 \%$ & $3,6 \%$ \\
$34-46$ años & $47,7 \%$ & $11,7 \%$ & $35,4 \%$ & $8,7 \%$ \\
Letras & $57,9 \%$ & $6,5 \%$ & $30,2 \%$ & $3,4 \%$ \\
Ciencias & $46,6 \%$ & $8,5 \%$ & $32,7 \%$ & $5,9 \%$ \\
Total & $52,6 \%$ & $7,2 \%$ & $31,4 \%$ & $4,3 \%$ \\
\hline
\end{tabular}

Fuente: Elaboración propia.

Si miramos el empleo de archivos de imagen, video o sonido, vemos que en general los sujetos con los que trabajamos, los emplean poco (cuadro 9). Y las diferencias en ninguna de las variables (sexo, edad, titulación) resultan llamativas. Aunque en la tabla anterior vemos cómo puntúan levemente más alto los hombres, los mayores y los titulados en "Ciencias".

Por último, los datos obtenidos en cuanto al uso de textismos o disortografías vemos que, en general nuestra muestra, los emplea poco. Y existen diferencias dentro de los grupos, con puntuaciones más elevadas en el caso de las mujeres, los jóvenes y los titulados en "letras". En el caso del sexo y del tipo de estudios, las diferencias son notables, mientras que las diferencias por edad son menos relevantes.

Cuadro 9. Porcentajes del uso de textismos en conversaciones e intervenciones por sexo, edad y titulación

\begin{tabular}{lccccc}
\hline & Palabras & InTERVENC. & TEXTISMOS & $\begin{array}{c}\text { \% PAlabRAS } \\
\text { CON TEXTISMOS }\end{array}$ & $\begin{array}{c}\text { \% INTERVENC. } \\
\text { CON TEXTISMOS }\end{array}$ \\
\hline Hombres & 10.841 & 1.869 & 1.438 & $13,30 \%$ & $76,90 \%$ \\
Mujeres & 9.562 & 2.003 & 2.155 & $22,50 \%$ & $107,60 \%$ \\
$23-33$ años & 17.098 & 3.343 & 3.213 & $18,80 \%$ & $96,10 \%$ \\
$34-46$ años & 3.306 & 529 & 380 & $11,50 \%$ & $71,80 \%$ \\
Letras & 12.913 & 2.491 & 2.585 & $20,00 \%$ & $103,80 \%$ \\
Ciencias & 7.491 & 1.381 & 1.008 & $13,50 \%$ & $73,00 \%$ \\
Total & 20.404 & 3.872 & 3.593 & $17,60 \%$ & $92,80 \%$ \\
\hline
\end{tabular}

Fuente: Elaboración propia. 


\section{Discusión}

Los resultados a los que hemos llegado, nos permiten abrir un diálogo en torno a diversos asuntos, aunque no puedan ser agotadas en estas líneas. Queremos destacar algunas cuestiones para seguir la reflexión científica en torno al uso de este medio digital que, en estos momentos, es la aplicación más utilizada en el mundo, por número de usuarios, entre los clientes de teléfonos inteligentes. Ofrecidos los resultados en el apartado anterior, vamos a hacer algunas comparaciones entre nuestros datos y los resultados de investigaciones similares.

En cuanto a la extensión de los textos, si comparamos estos resultados con los obtenidos por Vázquez-Cano, Mengual-Andrés y Roig-Vila (2015) sobre una muestra de adolescentes, comprobamos que las conversaciones de nuestros sujetos tienen menos intervenciones (ellos 20 nosotros 16) y menos palabras (ellos 12 y nosotros 5,2) por intervención. Por lo que se puede inferir, al menos en estos casos, que los adolescentes emplean mayor número de intervenciones ("hablan más") y se expresan con mayor número de palabras. Está ocurriendo lo contrario que hace unos años, donde los mensajes de texto a través de SMS, incorporaron al lenguaje digital un ahorro de palabras que en estos momentos no es necesario.

También es curioso observar cómo las conversaciones de las mujeres son más largas que las de los hombres, lo que podría contribuir a explicar las diferencias de tipo lingüístico que existen entre hombres y mujeres, tanto en la longitud del discurso como en su contenido (Mora, 2016). También hemos observado cómo economizan más, en cuanto al número de intervenciones, los mayores que los jóvenes, omitiendo frases innecesarias. En los estudiantes de "ciencias", ocurre algo similar, respecto a los de "letras", cosa, esta última, que podríamos considerar lógica dada la elección profesional que puede estimarse un indicador de la competencia lingüística que se presupone.

Nuestros resultados muestran un porcentaje de textismos en el $17,6 \%$ de las palabras frente al $37,2 \%$ de textismos localizados entre adolescentes en la investigación de Vázquez-Cano, Mengual-Andrés y Roig-Vila (2015). Diferencia muy significativa que apoyaría la idea de que a menor edad y/o nivel de estudios se percibe una mayor disonancia con las reglas de escritura tradicional. Algo similar, aunque con diferencias menos relevantes, ocurre con el uso de emoticonos que varía de $1,36 \%$ del total de palabras en esta investigación frente al 2,09\% de la muestra de adolescentes (VázquezCano, Mengual-Andrés y Roig-Vila, 2015). Disparidad que nos permite manifestar que, en estos casos, el empleo de emoticonos disminuye en relación al aumento de la edad y/o del nivel de estudios.

Las diferencias entre nuestra muestra y la de adolescentes en virtud de los archivos empleados son muy llamativas, dado que varían de $0,8 \%$ en esta investigación mientras que en el estudio de Vázquez-Cano, Mengual-Andrés y Roig-Vila (2015) sobre estudiantes de la ESO estos elementos llegan al 10,2\% en relación al número de palabras. Lo que nos permite afirmar, igual que en el caso de los emoticonos o los textismos que el aumento de la edad y del nivel de estudios disminuye la incorporación de archivos de audio, vídeo o imagen.

De otra parte, si comparamos estos resultados con una investigación anterior sobre estudiantes universitarios de $2^{\circ}$ curso de Maestro de Educación Infantil (Gómez y Gómez del Castillo, 2015) podemos comprobar que los resultados son algo más altos en 
la utilización de textismos, emoticonos y archivos, pero estas diferencias no resultan significativas en relación al actual estudio sobre titulados superiores.

Antes de terminar este apartado, quisiéramos hacer referencia al tipo de textismos más observados en los mensajes, y comprobamos, en la misma línea que lo hacen otras investigaciones (Gómez-Camacho y Gómez del Castillo, 2015), que los textismos que más se utilizan son los producidos por:

- Omisión de mayúsculas en nombres propio y después de punto y seguido.

- Omisión de tildes.

- Cambio de letras por su oralidad fonética.

- Juntar palabras.

- Faltas de ortografía realizadas conscientemente (ej. omisión de h).

- Empleo de palabras en otro idioma o inexistentes.

- Uso de mayúsculas para enfatizar.

- Omisión de letras, especialmente vocales.

Para finalizar, señalar que hemos podido comprobar que la escritura digital, en los mensajes de texto, está cambiando sustancialmente respecto a los SMS. Los textismos o variaciones orto-tipográficas, como las denominan algunos autores, es menor que hace unos años, sobre todo en el acortamiento de palabras, dado que las actuales herramientas de mensajería (WhatsApp, QQ, Wechat...) son prácticamente gratuitas y se puede escribir cuantos mensajes se deseen sin coste adicional. Esto está modificando considerablemente el tipo de escritura respecto al SMS de 140 caracteres y coste 0,15 céntimos muy utilizado hasta hace muy pocos años.

\section{Conclusiones}

Como conclusión de este trabajo, podemos afirmar que la comunicación por mensajería instantánea ha dejado de ser exclusivamente de tipo textual, y cada vez más el intercambio de información, opiniones o sentimientos se hace más expresiva, rica y variada con otros elementos comunicativos a través de imágenes, vídeos, audios, stickers o referencias a nuevas informaciones con enlaces a otras webs; este uso multi-mediático de la mensajería se ha impuesto.

La incorporación del teléfono móvil a los procesos de enseñanza-aprendizaje (m-learning) está siendo cada vez más importante, a veces por iniciativa del profesor y otras formando parte de los procesos de aprendizaje personales o grupales de los propios alumnos. De hecho, podemos afirmar que se está produciendo un cambio importante en el proceso educativo, de manera que el aprendizaje que utiliza como recurso importante los dispositivos móviles, generará nuevos enfoques en las concepciones pedagógicas en las que un elemento clave será el fomento de la investigación y el autoaprendizaje en el estudiante.

No se puede negar que WhatsApp es la herramienta comunicativa más importante de nuestro entorno en estos momentos, usada por el 100\% de la muestra, y que está revolucionando la comunicación entre individuos o grupos de personas. Además, esta 
herramienta comunicacional es seguida por sujetos de todas las edades, es decir, no solo es usada mayoritariamente por los jóvenes (como era el caso de los SMS), sino que también los adultos son muy dados a este tipo de aplicación (Padrón, 2013). Y, por tanto, se abre un abanico de posibilidades de cara a la comunicación entre alumnos, profesores, familiares... que hasta ahora no existía.

Los usuarios más jóvenes realizan conversaciones más largas que los más mayores, con más intervenciones y más palabras; y en muchos casos prefieren la mediación del WhatsApp que la llamada telefónica tradicional o la comunicación presencial. También hemos comprobado que las mujeres realizan conversaciones más largas que los hombres, pero no se han encontrado diferencias notables entre los sujetos de titulaciones de "letras" o de "ciencias". En el ámbito educativo, esto supone una nueva oportunidad para trabajar la competencia lingüística.

El uso de textismos y las disonancias con la norma culta de escritura se dan más frecuentemente entre los sujetos más jóvenes y/o con menor nivel de estudios que en los graduados. Lo mismo ocurre con el uso de archivos de audio, video o imágenes intercalados en la conversación a través de WhatsApp y con la utilización de emoticonos.

Nuestro estudio corrobora que la norma digital de SMS antes, o actualmente de WhatsApp, no amenaza ni perjudica la forma de escritura tradicional característica de los hablantes cultos en castellano; al contrario, las disortografías que se utilizan de los mensajes de texto y la incorporación de los recursos multimedia en la escritura están creando una nueva norma de comunicación.

Desde una perspectiva formativa, la alfabetización digital en la comunicación mediada por los teléfonos inteligentes resulta imprescindible entre los objetivos de la enseñanza, que no debe quedarse al margen de esta realidad. Los resultados de nuestro estudio demuestran que la totalidad de los graduados alternan entre la norma de escritura culta utilizada habitualmente en los trabajos académicos con una norma disortográfica; reiterando que "la condena generalizada de estos textos o su exclusión del currículo resulta incomprensible y contraproducente" (Gómez-Camacho y Gómez-del-Castillo, 2015, p. 112).

La muestra de hablantes de posgrado se caracteriza por estar más próximos a la norma académica culta del español que otros colectivos más jóvenes o con niveles de estudios inferiores, aunque mantiene los rasgos distintivos de multimodalidad y discrepancias ortográficas, que se utilizan limitadas a determinados géneros de comunicación on-line, como las conversaciones con la aplicación WhatsApp.

Creemos que el número de sujetos que componen la muestra de nuestro estudio podría haber sido más amplio, bien porque hubiesen participado más estudiantes del Máster de Secundaria, bien porque hubiésemos trabajado con otros titulados tomados de otras instituciones o de la población general. Para trabajos futuros habría que tener en cuenta esta circunstancia, pudiendo replicar la investigación con otros sujetos de características sociales, culturales, de género o edad diferentes. También se podría ampliar este tipo de estudio al análisis de contenido de las conversaciones mantenidas tanto en contextos profesionales como en contextos informales. 


\section{Referencias}

Bernicot, J., Goumi, A., Bert-Erboul, A. y Volckaert-Legrier, O. (2014). How do skilled and lessskilled spellers write text messages? A longitudinal study. Journal of Computer Assisted Learning, 30(6), 559-576. https://doi.org/10.1111/jcal.12064

Blanch, M., Betancort, S. y Martínez, M. (2016). El videoblog en el aula de lengua y literatura de secundaria. Una propuesta práctica. REICE. Revista Iberoamericana sobre Calidad, Eficacia y Cambio en Educación, 14(3), 33-49. https://doi.org/10.15366/reice2016.14.3.002

Bouhnik, D. y Deshen, M. (2014). WhatsApp goes to school: Mobile instant messaging between teachers and students. Journal of Information Technology Education Research, 13, 217-231.

Calero, M. L. (2014). El discurso de WhatsApp: Entre el Messenger y el SMS. Oralia, 17, 85-114.

Capilla, E. y Cubo, S. (2017). Phubbing. Conectados a la red y desconectados de la realidad. Un análisis en relación al bienestar psicológico. Púxel-Bit. Revista de Medios y Educación, 50, 173-185.

Clairá, A. (2013). ¿Conectados o comunicados? Recuperado de http://www.ciudadnueva.org.ar/revista/ 541/sociedad/conectados-o-comunicados

Cremades, R., Maqueda, E. y Onieva, J. L. (2016). Posibilidades didácticas de la escritura digital ubicua en la aplicación WhatsApp messenger. Revista Letral, 16, 106-120.

Díaz-Jatuf, J. (mayo, 2014). El WhatsApp como herramienta de intervención didáctica para fomentar el aprendizaje cooperativo. Comunicación presentada en las X Jornadas de material didáctico y experiencias innovadoras en educación superior. Buenos Aires: Universidad de Buenos Aires.

Galán, C. (2011). Homo loquens, homo virtualis. Revista de Estudios de Juventud, 93, 11-26.

Gann, R., Bartoszuk, K. y Anderson, J. (2010). If u txt 2 much, duz it mean u cant spell: Exploring the connection between SMS use and lowered performance in spelling. The International Journal of the Book, 7(2), 69-77. https://doi.org/10.18848/14479516/CGP/v07io2/36807

Giménez, M. y Zirpoli, R. (abril, 2015). Trastornos psicológicos vinculados al uso de WhatsApp. Comunicación presentada en el VII Congreso Internacional de Investigación y Práctica Profesional en Psicología XXII Jornadas de Investigación. Buenos Aires: Universidad de Buenos Aires.

Gómez-Camacho, A. y Gómez-del-Castillo, M.T. (2013). Desarrollo de las competencias lingüística y digital a través de las redes sociales. En S. Castillo (Coord.), Reflexiones, análisis y propuestas sobre la formación del profesorado en educación secundaria (pp. 285-288). Madrid: UNED.

Gómez-Camacho, A. y Gómez-del-Castillo, M. T. (2015). Escritura ortográfica y mensajes de texto en estudiantes universitarios. Perfiles Educativos, 37(150), 91-104.

Han, T. y Keskin, F. (2016). Using a mobile application (WhatsApp) to reduce EFL speaking anxiety. Gist Education and Learning Research Journal, 12, 29-50.

Hernández, D. (2016). WhatsApp se corona superando los 1.000 millones de usuarios. Recuperado en http://computerhoy.com/noticias/apps/WhatsApp-corona-superando-1000-millonesusuarios-39761

Iniciarte, M. (2004). Tecnologías de la información y la comunicación. Un eje transversal para el logro de aprendizajes significativos. REICE. Revista Iberoamericana sobre Calidad, Eficacia y Cambio en Educación, 2(1), 1-29. 
López, F. (2002). El análisis de contenido como método de investigación. XXI, Revista de Educación, 4, 167-179.

Martínez-Parejo, R. (2016). Desarrollo de la competencia escrita en la enseñanza de lenguas extranjeras a través del uso de dispositivos móviles. Revista Complutense de Educación, 27(2), 779-803. https://doi.org/10.5209/rev_RCED.2016.v27.n2.48317

Mora, F. (2016). Cuando el cerebro juega con las ideas. Madrid: Alianza.

Morduchowicz, R. (2013). Los adolescentes del siglo XXI. Ciudad de México: Fondo de Cultura Económica.

Oliveira, A., Versuti, A. y Vaz, W. F. (2016). Perspectivas para o uso do WhatsApp no estímulo à aprendizagem dos sujeitos. Práticas Pedagógicas para Inclusão e Diversidade, 1(7), 222-236.

Padrón, C. J. (2013). Estrategias didácticas basadas en aplicaciones de mensajería instantánea WhatsApp exclusivamente para móviles (mobile learning) y el uso de la herramienta para promover el aprendizaje colaborativo. Eduweb, 7(2), 123-134.

Powell, D. y Dixon, M. (2011). Does SMS text messaging help or harm adults' knowledge of standard spelling? Journal of Computer Assisted Learning, 27(1), 58-66. https://doi.org/10.1111/j.1365-2729.2010.00403.x

Rubio, J. y Perlado, M. (2015). El fenómeno WhatsApp en el contexto de la comunicación personal: Una aproximación a través de los jóvenes universitarios. Icono 14(13), 73-94. https://doi.org/10.7195/ri14.v13i2.818

Sampietro, A. (2016). Emoticonos y multimodalidad. El uso del pulgar hacia arriba en WhatsApp. Aposta: Revista de Ciencias Sociales, 69, 271-295.

Valencia, Y. y García, V. (2010), La escritura simbólica y el lenguaje escrito en los usuarios de Messenger. Comunicar, 34, 155-162. https://doi.org/10.3916/C34-2010-03-15

Vázquez-Cano, E., Mengual-Andrés, S. y Roig-Vila, R. (2015). Análisis lexicométrico de la especificidad de la escritura digital del adolescente en WhatsApp. Revista de Lingüística Teórica y Aplicada, 53(1), 83-105. https://doi.org/10.4067/S07 18-48832015000100005

Vela, C. y Cantamutto, L. (2016). De participante a observador: el método etnográfico en el analisis de las interacciones digitales de WhatsApp. Tonos Digital, 31, 1-22.

Villamandos, M. (2016). 10 cifras para entender WhatsApp y sus descomunales dimensiones. Recuperado de http://www.actualidadgadget.com/10-cifras-para-entender-WhatsApp/

\section{Breve CV de la autora}

\section{María-Teresa Gómez-del-Castillo}

Profesora Contratada Doctora Interina en la Universidad de Sevilla, 5 años de experiencia en la Facultad de Ciencias de la Educación. Cuenta con 16 años de experiencia como profesora titular de las titulaciones de Magisterio y Psicopedagogía en el CES Cardenal Spínola. Es miembro del Grupo de Investigación GID desde el año 2001. Defendió la tesis en el año 2001 titulada "Análisis de valores en el software educativo multimedia” dirigida Dr. Julio Cabero Almenara. Sus líneas actuales de investigación son: Formación de profesorado, TIC y Educación y diversidad y TIC. ORCID ID: OOOO-OOO2-1320-6644. Email: mgomezdelcastillo@us.es 